\title{
Langmuir
}

pubs.acs.org/Langmuir

(c) XXXX American Chemical Society

\section{Structure and Morphology of Thin Films of Linear Aliphatic Polyesters Prepared by Spin-Coating}

\author{
J. J. Hernández, D. R. Rueda, * M. C. García-Gutiérrez, A. Nogales, and T. A. Ezquerra \\ Instituto de Estructura de la Materia, CSIC, Serrano 121, 28006 Madrid, Spain \\ M. Soccio, N. Lotti, and A. Munari \\ Dipartamento di Chimica Applicata e Scienza dei Materiali, Universitá di Bologna, Viale Risorgimento 2, \\ 40126 Bologna, Italy
}

Received February 2, 2010. Revised Manuscript Received March 30, 2010

\begin{abstract}
Thin films, with thicknesses from 10 to $400 \mathrm{~nm}$ of linear aliphatic polyesters $(X, Y)$, based on propylenediol $(X=3)$ and on dicarboxylic acid of different chain length $\left(Y=2,3\right.$, and $4 \mathrm{CH}_{2}$ units) were prepared by spin coating of $\mathrm{CHCl}_{3}$ polymer solutions with different polymer concentrations. Morphology and structure of the spin coated thin films were investigated by atomic force microscopy (AFM) and by grazing incidence X-ray scattering techniques at small, (GISAXS) and wide angles (GIWAXS). AFM revealed a strong dewetting for all three polymers for coatings thinner than $100 \mathrm{~nm}$. The polymer films are clearly semicrystalline for thicknesses higher than $50 \mathrm{~nm}$. GIWAXS of the thicker films revealed their oriented crystalline nature. An edge-on-lamellae morphology is clearly shown by the AFM-phase images even for relatively thin films. SAXS with the beam parallel to the sample plane also support the presence of lamellae perpendicular to the substrate. The use of a $\mu$-beam helped to interpret the GIWAXS patterns and allowed to obtain oriented WAXS patterns from melt solidified filaments. Thus, a crystal chain packing is proposed for the three polymers and consequently the indexing of the observed reflections. Accordingly, the polymer chains lie parallel to the substrate being the $b c$ plane of the monoclinic crystal unit cell parallel to the substrate.
\end{abstract}

\section{Introduction}

The synthesis and characterization of new biodegradable polymers is a topic of increasing interest mainly due to their potential applications in waste treatment. Besides environmental protection and recycling, biodegradable polymers can be used in wound closures, orthopedic implants, drug delivery systems, and matrices for cell culture among other applications. ${ }^{1-4}$ The preparation of thin and ultrathin polymer films is essential for some of these developments. ${ }^{3,4}$ In general, polymer ultrathin films can exhibit different structure and properties than either thick films or bulk materials. The knowledge of the mechanism and the underlying forces involved in polymer dewetting is crucial to predict stability conditions of the films for practical use..$^{5-7}$ Additionally, thin polymer films introduce two-dimensional spatial confinement which may affect the molecular order, ${ }^{8}$ the crystallization

*Corresponding author.

(1) Langer, R.; Tirrell, D. A. Nature 2004, 428 (6982), 487-492.

(2) Papageorgiou, G. Z.; Vassiliou, A. A.; Karavelidis, V. D.; Koumbis, A.; Bikiaris, D. N. Macromolecules 2008, 41, 1675-1684.

(3) Campillo-Fernandez, A. J.; Pastor, S.; Abad-Collado, M.; Bataille, L.; Gomez-Ribelles, J. L.; Meseguer-Duenas, J. M.; Monleon-Pradas, M.; Artola, A.; Alio, J. L.; Ruiz-Moreno, J. M. Biomacromolecules 2007, 8, 2429-2436.

(4) Loschonsky, S.; Shroff, K.; Worz, A.; Prucker, O.; Ruhe, J.; Biesalski, M. Biomacromolecules 2008, 9, 543-552.

(5) Segalman, R. A.; Green, P. F. Macromolecules 1999, 32, 801-807.

(6) Seemann, R.; Herminghaus, S.; Jacobs, K. Phys. Rev. Lett. 2001, 86, 5534--.

(7) Seemann, R.; Herminghaus, S.; Neto, C.; Schlagowski, S.; Podzimek, D.; Konrad, R.; Mantz, H.; Jacobs, K. J. Phys.: Condens. Matter 2005, 17, S267-S290.

(8) Rueda, D. R.; Nogales, A.; Hernandez, J. J.; Garcia-Gutierrez, M. C.; Ezquerra, T. A.; Roth, S. V.; Zolotukhin, M. G.; Serna, R. Langmuir 2007, 23, 12677-12681.

(9) Capitan, M. J.; Rueda, D. R.; Ezquerra, T. A. Macromolecules 2004, 37, 5653-5659.

(10) Ma, Y.; Hu, W. B.; Reiter, G. Macromolecules 2006, 39, 5159-5164.

(11) Wang, Y.; Chan, C. M.; Ng, K. M.; Li, L. Macromolecules 2008, 41, $2548-2553$. behavior, ${ }^{9,10}$ the morphology, ${ }^{11}$ or even the crystalline structure ${ }^{12}$ of the polymer. One important aspect in thin films of semicrystalline polymers is related to the orientation of the crystalline lamellae. Although in general it has been observed that thin films of several hundreds of nanometers exhibit predominantly edge-on lamellae and ultrathin films exhibit flat-on lamellae there are several factors, besides thickness, like the interaction with the substrate ${ }^{10}$ or the crystallization temperature ${ }^{11}$ that may control the relative abundance of different crystalline lamellae orientations. Monte Carlo simulations suggest that for polymers deposited over slippery, nonattracting walls, the edge-on lamellar crystal morphology would be the more favorable while flat-on lamellae would be more abundant when the substrate is sticky. ${ }^{10}$ Among synthetic polymers, aliphatic polyesters are very attractive because they may combine properties such as biocompatibily and biodegradablility with physical and chemical characteristics similar to those of commodity polymers. ${ }^{13-15}$ In particular, polyesters based on 1,3-propanediol and aliphatic dicarboxylic acids have attracted some interest due to the fact that they can be prepared with different chain unit length and high molecular weight. ${ }^{15}$ In addition 1,3-propanediol prepared by fermentation from renewable resources is commercially available at relative low cost. Although bulk properties of these polymers have been studied ${ }^{15-17}$

(12) Park, Y. J.; Kang, S. J.; Lotz, B.; Brinkmann, M.; Thierry, A.; Kim, K. J.; Park, C. Macromolecules 2008, 41, 8648-8654.

(13) Martinez-Palau, M.; Franco, L.; Puiggali, J. Polymer 2007, 48, 6018-6028.

(14) Gesti, S.; Zanetti, M.; Lazzari, M.; Franco, L.; Puiggali, J. Eur. Polym. J. 2009, 45, 398-409.

(15) Soccio, M.; Lotti, N.; Finelli, L.; Gazzano, M.; Munari, A. Polymer 2007, $48,3125-3136$

(16) Soccio, M.; Nogales, A.; Lotti, N.; Munari, A.; Ezquerra, T. A. Polymer 2007, 48, 4742-4750.

(17) Soccio, M.; Nogales, A.; Lotti, N.; Munari, A.; Ezquerra, T. A. Phys. Rev. Lett. 2007, 98 (3). 
not much information about the structure and morphology of their thin and ultrathin films has been reported. In this work we present data on the structure and morphology of a series of thin and ultrathin films of linear aliphatic polyesters including poly(propylene succinate) (PPS), poly(propylene glutarate) (PPG), and poly(propylene adipate) (PPA) prepared by spin-coating over silicon substrates. We have combined experiments in the real space by means of atomic force microscopy (AFM) and in the reciprocal space by means of X-ray scattering. Lamellar crystal edge-on morphology seems to be a rather robust characteristic of the investigated aliphatic polyester thin films.

\section{Experimental Section}

2.1. Materials and Samples Preparation. Poly(propylene succinate) (PPS), poly(propylene glutarate) (PPG), and poly(propylene adipate) (PPA) were synthesized by using the twostages polycondensation procedure as previously reported. ${ }^{15}$ These polymers are semicrystalline and their monomeric units are shown in the inset of Figure 1. Melting and glass transition temperatures as determined by differential scanning calorimetry (DSC) are reported in Table 1. As one can see these polymers are characterized by low melting temperatures and glass transitions below room temperature.

Thin polymer films were prepared by spin coating a solution of the polymer in chloroform $\left(\mathrm{CHCl}_{3}\right)$ on silicon wafers (about $19 \times$ $25 \mathrm{~mm}^{2}$ ) in the following way. First, a polymer mother solution $(40 \mathrm{~g} / \mathrm{L})$, referred hereafter as (1:0), was prepared. From this other less concentrated solutions were obtained by selective dilution. In particular, the following polymer solutions were prepared: $20 \mathrm{~g} / \mathrm{L}$ (1:1), 13.3 g/L (1:2), $8 \mathrm{~g} / \mathrm{L}(1: 4), 4.4 \mathrm{~g} / \mathrm{L}(1: 8), 2.3 \mathrm{~g} / \mathrm{L}(1: 16), 1.2 \mathrm{~g} /$ $\mathrm{L}(1: 32)$, and $0.6 \mathrm{~g} / \mathrm{L}(1: 64)$. Second, by means of a syringe, $0.1 \mathrm{~mL}$ of a given solution was instantly dropped on the center of a silicon (100) substrate rotating at a speed of $1730 \mathrm{rpm}$. Silicon wafers were obtained from Wafer World, Inc. They had both surfaces polished and were used as supplied with its native silicon oxide surface of $\approx 2 \mathrm{~nm}$ in thickness as determined by ellipsometry, ${ }^{8}$ after cleaning with soap solution (commercial detergent: Procter \& Gamble) and bidistilled water. Occasionally, polymer coatings were also prepared on cover glass substrates for comparison purposes. The thickness of the polymer films as derived combining ellipsometry, imaging ellipsometry and atomic force microscopy measurements increases with polymer concentration within the 10-400 $\mathrm{nm}$ range (see Figure 1).

2.2. Atomic Force Microscopy. A Nanoscope IIIA Multimode from Veeco operating in the tapping mode was used to investigate the surface morphology of the spin coated films at room temperature. AFM images were conveniently analyzed by means of the software supplied. Thus, morphological magnitudes like the power spectral density and average roughness were measured for each sample. The calculation of the power spectral density function (PSD) was accomplished by the software supplied by Veeco. PSD is calculated from the AFM pictures by a two-dimensional (2D) Fourier transformation and a radial average of the isotropic Fourier space data. ${ }^{18,19}$

2.3. X-ray Scattering. 2.3.1. Grazing Incidence X-ray Scattering. Grazing incidence X-ray scattering both at small angle (GISAXS) and at wide angle (GIWAXS) were accomplished by using synchrotron radiation in the BW4 beamline at HASYLAB (Hamburg, Germany) and in the ID01 beamline at ESRF (Grenoble, France), respectively. Grazing incidence X-ray scattering (GIXS) has been revealed as a powerful technique for the investigation of the structure developed in polymer thin

(18) Bollinne, C.; Cuenot, S.; Nysten, B.; Jonas, A. M. Eur. Phys. J. E 2003, 12, 389-395.

(19) Bauer, E.; Maurer, E.; Mehaddene, T.; Roth, S. V.; Muller-Buschbaum, P. Macromolecules 2006, 39, 5087-5094.

(20) Busch, P.; Posselt, D.; Smilgies, D. M.; Rheinlander, B.; Kremer, F.; Papadakis, C. M. Macromolecules 2003, 36, 8717-8727.

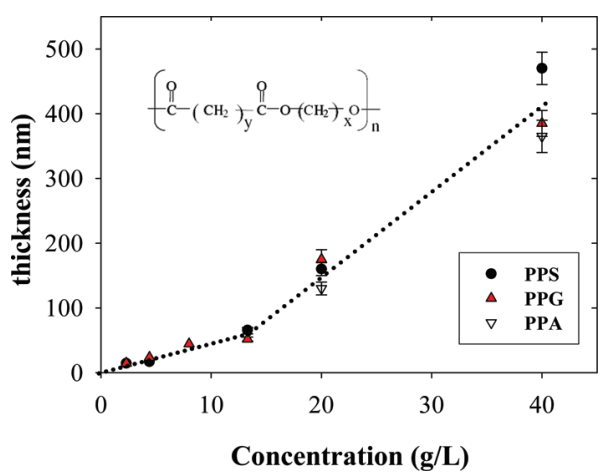

Figure 1. Thickness values of the spin-coated polymer films as a function of the polymer solution concentration for the different polymers: $(\bullet)$ PPS, $(\Delta)$ PPG, and $(\nabla)$ PPA. Inset: Monomeric units of the poly(propylene dicarboxylate)s investigated, where $x=3$ and $y=2$ for poly(propylene succinate) (PPS), $y=3$ for poly(propylene glutarate) (PPG), and $y=4$ for poly(propylene adipate) (PPA).

Table 1. Molecular and Thermal Characterization Data ${ }^{15}$

\begin{tabular}{lcrcc}
\hline polymer & $M_{\mathrm{n}}$ & \multicolumn{1}{c}{$M_{\mathrm{w}}$} & $T_{\mathrm{m}}\left({ }^{\circ} \mathrm{C}\right)^{a}$ & $T_{\mathrm{g}}\left({ }^{\circ} \mathrm{C}\right)^{b}$ \\
\hline PPS & 36400 & 79000 & 44 & -29 \\
PPG & 43500 & 100000 & 54 & -49 \\
PPA & 31200 & 75500 & 40 & -54
\end{tabular}

${ }^{a}$ Semicrystalline sample, first DSC scan of the as synthesized sample. ${ }^{b}$ Amorphous sample, second DSC scan after melt quenching.

films. ${ }^{8,19-23}$ For GISAXS experiments a wavelength, $\lambda=0.1381$ $\mathrm{nm}$, a beam-size of $50 \mu \mathrm{m}$, a Mar CCD camera $(2048 \times 2048$ pixels) and a pixel size of $79.1 \mu \mathrm{m}$, and a sample-to-detector distance of $2.240 \mathrm{~m}$ were used. The incidence angle $\alpha_{i}\left(0.4^{\circ}\right)$ was selected to be twice the critical angle for the silicon substrate which is larger than that one for polymer materials. Thus the GISAXS patterns concern the whole thickness of the polymer film. Exposure times from $20 \mathrm{~s}$ up to $300 \mathrm{~s}$ were used to get the GISAXS patterns for the different samples investigated. For GIWAXS experiments an energy beam of $10 \mathrm{keV}(\lambda=0.124 \mathrm{~nm})$ and a Princeton detector $(1339 \times 1299$ pixels $)$ with a pixel area of $48.17 \times 49.04 \mu \mathrm{m}^{2}$ were used. The detector plane was maintained tilted $22^{\circ}$ from vertical position and located at $0.067 \mathrm{~m}$ of the sample center. GIWAXS patterns were recorded at different $\alpha_{i}$ values ranging from $0.36^{\circ}$ to $0.06^{\circ}$. Calibration was done by using a standard of $\mathrm{Al}_{2} \mathrm{O}_{3}$ and also checked by using a sample of Lupolen. GIWAXS images were properly corrected considering the geometry of the experiment at ID01. ${ }^{24}$ GIWAXS patterns of the Si substrate were also recorded at different grazing incidence angles. The GIWAXS pattern of each sample was conveniently subtracted by the corresponding one of the Si substrate taking into account the different intensity observed at the meridian and close to the beam stop. Both GISAXS and GIWAXS patterns were analyzed by using the Fit2D software.

2.3.2. X-ray Scattering with Grazing Incidence Configuration at $\alpha_{i}=0$. Experiments were additionally performed in BW4 (HASYLAB) and in the microfocus beamline at ESRF (ID13), respectively by using the GIXS configuration with an incidence angle $\alpha_{i}=0$. At ID13 a beam size of $1 \mu \mathrm{m}$ was used. As it will be shown in this paper, under these conditions valuable information, not accessible by GISAXS, about the nanostructure of the polymer film can be obtained.

(21) Müller-Buschbaum, P. Lecture Notes in Physics: Applications of Synchrtron Light to Scattering and Diffraction in Materials and Life Sciences; Springer: Berlin, 2009; Vol. 776, p 61

(22) Muller-Buschbaum, P. Prog. Colloid Polym. Sci. 2006, 132, 23-32.

(23) Yoneda, Y. Phys. Rev. 1963, 131, 2010.

(24) Boesecke, P. J. Appl. Crystallogr. 2007, 40, S423-S427. 


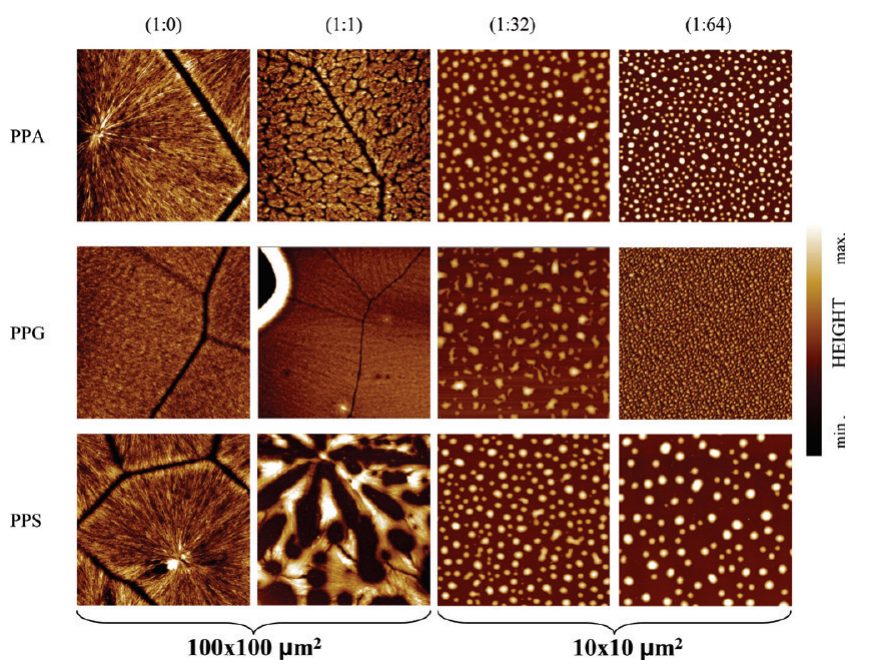

Figure 2. Height AFM images for selected polymer films prepared, from left to right, from the $(1: 0),(1: 1),(1: 32)$, and $(1: 64)$ polymer solutions.

2.3.3. Wide Angle X-ray Scattering. In addition, the microfocus beam of ID13 beamline at ESRF was used to investigate the bulk crystalline structure of thin filaments of the polymers obtained by melt drawing and further solidification on Kapton foils.

\section{Results and Discussion}

3.1. Morphology of Polymer Thin Films As Revealed by AFM. Figure 2 shows AFM height images for the thin films prepared from the (1:0), (1:1), (1:32), and (1:64) solutions. The thicker films, (1:0), reveal at this magnification the presence of big spherulites which are of the order of $10^{2} \mu \mathrm{m}$ in size. Clear dewetting effects appear for thinner samples prepared from solutions more diluted than (1:1). This indicates that films thinner than $\approx 100 \mathrm{~nm}$ are not stable and tend to dewet on substrates of silicon covered with native silicon oxide. This observation suggests these substrates to have a nonattractive, slippery, character otherwise dewetting would be prevented. ${ }^{10}$ The semicrystalline nature of the polymer films is revealed by the presence of crystalline lamellae as shown in the selected AFM phase images of Figure 3. Even samples where dewetting effects are significant exhibit a semicrystalline texture. Lamellar crystals are observed in all samples where droplet formation is not dominant like those prepared from solutions less diluted than (1:16). The AFM images reveal a characteristic semicrystalline morphology rich in edge-on lamellae. Very similar morphology to that shown in Figure 2 was observed for the three polymers prepared, under similar conditions, by using cover glass plates instead of Si wafers as substrate.

3.2. Structural Features As Revealed by X-ray Scattering Experiments. 3.2.1. Grazing Incidence X-ray Scattering at Small and Wide Angles. GISAXS measurements were accomplished in all the spin coated films. Only those films prepared from the more diluted solutions with well-defined droplet morphology exhibited out-of-meridian scattering signals. As an example, Figure 4a shows in the inset the GISAXS pattern of a PPA film prepared from the (1:64) solution. In this case, two resolved scattering maxima out of the meridian are clearly visible in the pattern over the strong diffuse intensity contribution known as Yoneda peak. ${ }^{23}$ This is illustrated in the corresponding intensity profile across the horizontal direction as shown in Figure 4a. Figure 5a shows in a common plot the power spectral density function derived from the AFM image of the PPA (1:64)

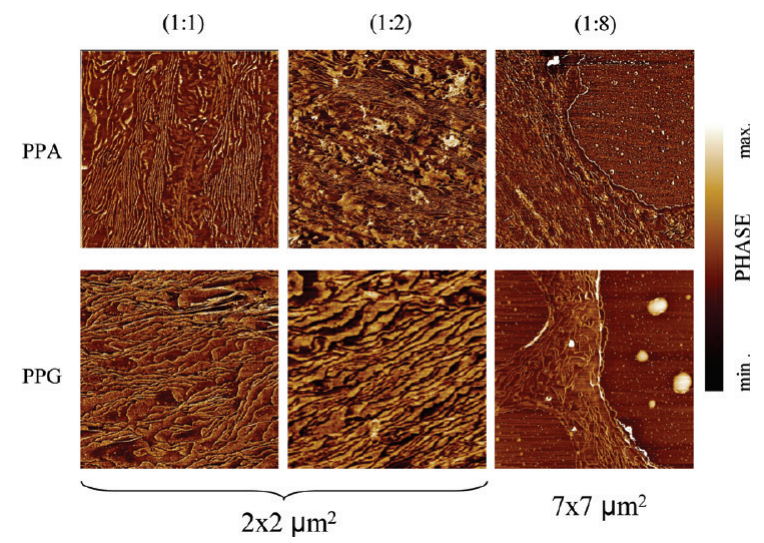

Figure 3. Phase AFM images for selected polymer films prepared, from left to right, from the $(1: 1),(1: 2)$, and (1:8) polymer solutions.

PPA
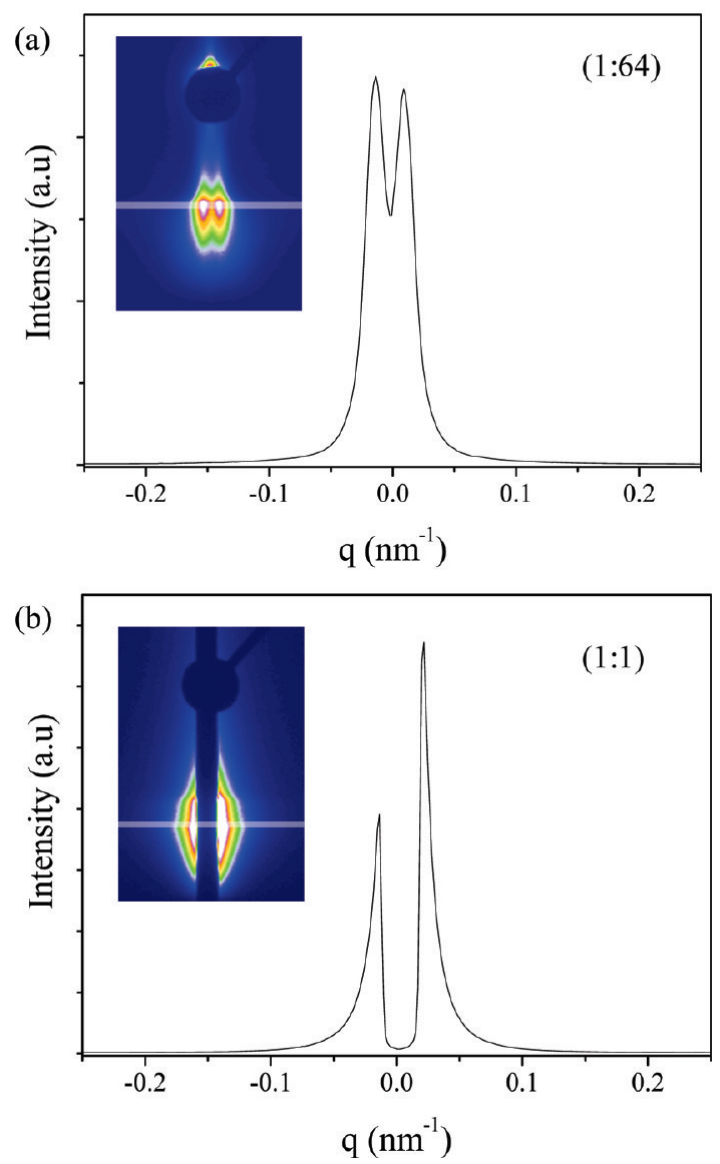

Figure 4. GISAXS patterns (insets) and corresponding y-cuts intensity profiles at $q_{z}=0.470 \mathrm{~nm}^{-1}\left(\alpha_{\mathrm{f}}=0.192^{\circ}\right)$ for (a) PPA $(1: 64)$ and (b) PPA $(1: 1)$.

sample and from the GISAXS scattering intensity. ${ }^{18,19}$ Both plots exhibit a maximum at a very similar scattering vector. The GISAXS correlation length derived from the maximum of the $y$-cut profile, $D_{y}\left(D_{y}=2 \pi / q_{y}^{\max } \approx 520 \mathrm{~nm}\right)$ is in rather good agreement with that derived from the PSD function $(505 \mathrm{~nm})$ as frequently observed in thin films containing homogeneous correlated motives. ${ }^{19}$ GISAXS measurements on thicker films exhibit a complete absence of out-of-meridian scattering signals. As an example, we show in Figure $4 b$ the GISAXS pattern of a PPA 

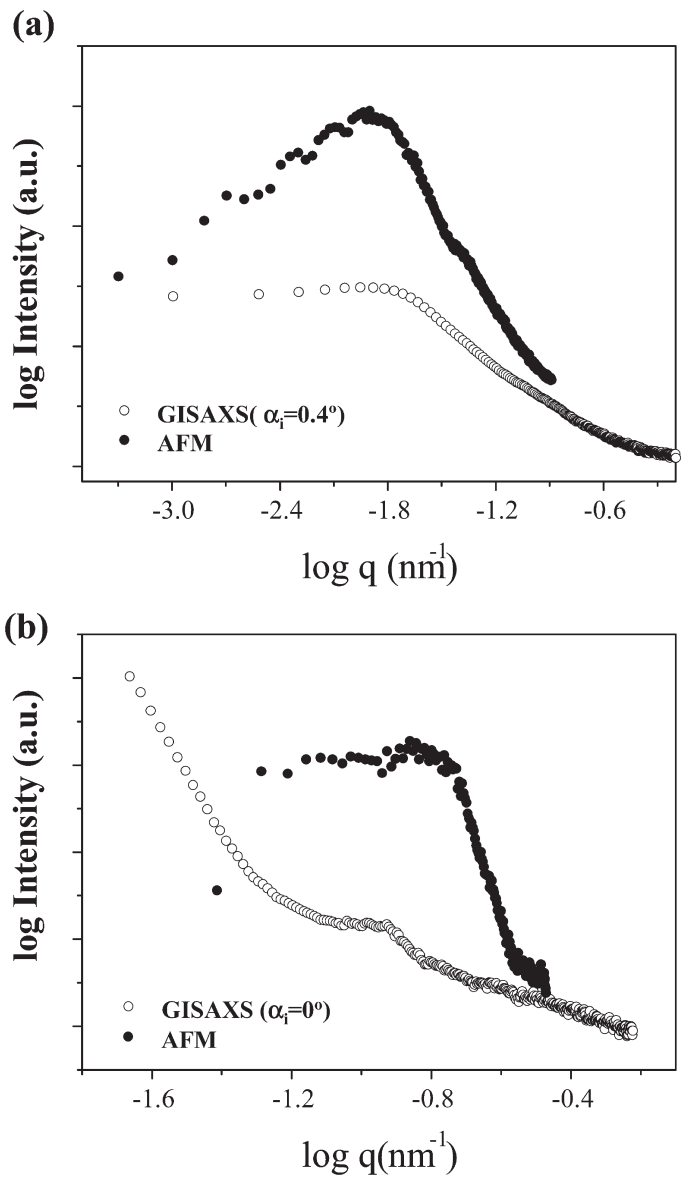

Figure 5. Comparison between the power spectral density function obtained from AFM images and the horizontal scattering intensity profiles obtained (a) by GISAXS ( $y$-cut at $q_{z}=0.470$ $\mathrm{nm}^{-1}$ ) for PPA $(1: 64)$ and (b) by GISAXS with $\alpha_{i}=0^{\circ}$ for PPA $(1: 1)$

(1:1) sample and its corresponding intensity profile across the horizontal direction. The continuous scattering along the meridian was so strong that a linear beamstop was required. At first glance this is a surprising result since the AFM images of the thicker films (Figure 2 and 3 ) do exhibit the presence of crystalline lamellae. The absence of scattering maxima out of the meridian could be due to a heterogeneous distribution of crystalline lamellar stacks. Although AFM essentially provides information about the film surface it is likely the existence of polymer crystals across the film thickness. Therefore some scattering at small angles should be expected. The semicrystalline nature of the thicker films, (1:0) and (1:1) has been verified by the GIWAXS experiments. In Figure 6a, we have presented selected GIWAXS patterns taken at $\alpha_{i}=0.12^{\circ}$ for the thickest (1:0) samples of PPA, PPG, and PPS. For samples (1:1) the GIWAXS patterns were very similar. General features of GIWAXS patterns do not vary at the different $\alpha_{i}$ values. In general, a GIWAXS pattern is equivalent to the half of a WAXS-pattern whose equator and meridian lines are parallel and perpendicular to the surface film plane. Thus, reflections on the equator will correspond to the scattering of vertical lattice planes. Patterns of Figure 6a clearly revealed the crystalline nature of these thin polymer films as well as some crystal orientation. Indexing of the observed reflections should reveal information about polymer chain orientation in the films. This is a difficult task mainly for two reasons. First, reflections in the GIWAXS patterns look to exhibit peak multiplicity. This means that a given $h k l$-reflection splits into several intensity (a)

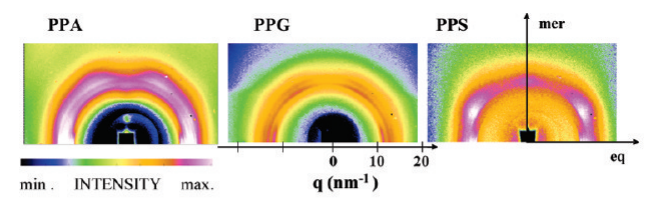

(b)

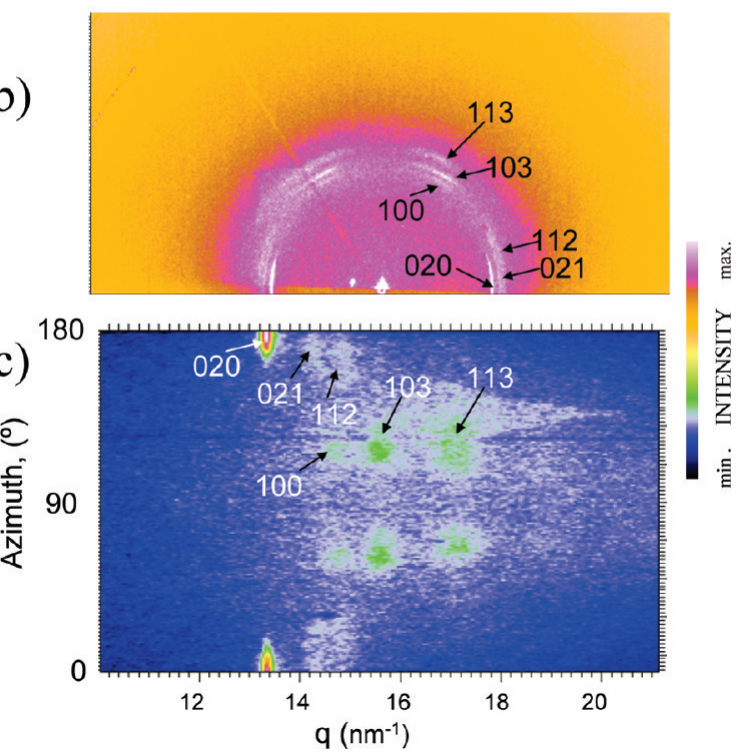

Figure 6. (a) GIWAXS patterns $\left(\alpha_{i}=0.12^{\circ}\right)$ for the thickest (1:0) sample of the three aliphatic polyesters investigated. The axes for the equator and meridian lines of the GIWAXS pattern were also indicated. (b) GIWAXS pattern with $\alpha_{i}=0^{\circ}$ obtained with a micro beam at the ID13 beamline at the ESRF from the spin coated sample PPA (1:0). (c) Projection corresponding to an interval of the scattering vector $q$ between 10 and $21 \mathrm{~nm}^{-1}$ Indices $h \mathrm{kl}$ of reflections are indicated in both two pictures.

maxima. This effect appears by the spreading of crystallites on the large illuminated area. ${ }^{25}$ In order to overcome this problem we performed X-ray experiments with grazing incidence configuration at $\alpha_{i}=0$ and with a smaller beam $($ of $\approx 1 \mu \mathrm{m})$ in order to reduce the illuminated area. The results will be presented in the next paragraph. Second, to our knowledge there are not crystal unit cell parameters available for the three polymers investigated here. To deal with this we accomplished X-ray experiments in polymer drawn filaments in an attempt to obtain additional crystal information. The results will be presented in section 3.2.3.

3.2.2. X-ray Scattering with Grazing Incidence Configuration at $\alpha_{i}=0$. Given the absence of structural information provided by GISAXS, additional experiments were performed by using the GIXS configuration but with an incidence angle $\alpha_{i}=0$. These experiments can be considered equivalent to those performed by SAXS and WAXS (in transmission geometry) but with the film surface parallel to the beam instead of being perpendicular. Since this experimental approach is, to the best of our knowledge, novel several aspects have to be discussed before proceeding. In principle, the X-ray beam with an incidence angle $\alpha_{i}=0$ placed above the sample surface would produce an evanescent wave touching the surface and thereby probing the surface structure only. To avoid this, the sample was located in such a way that the beam illuminated its whole thickness. This was possible because the beam size was always bigger than the thickness of the investigated samples. As described by MüllerBuschbaum $^{22}$ for grazing incidences $\alpha_{i} \approx 0$, a specular peak should appear below the Yoneda wing. In our case, the patterns

(25) Meyer, A. Ph.D. Thesis. University of Hamburg, 1998 
(a)

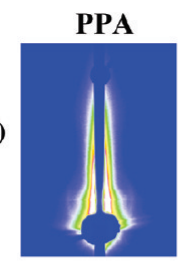

(b)

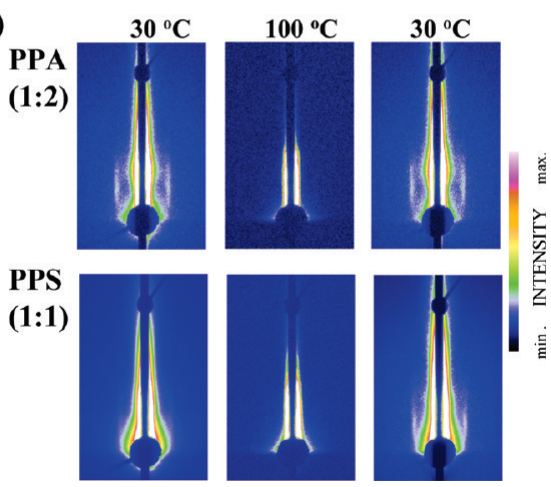

Figure 7. (a) GISAXS patterns with $\alpha_{i}=0^{\circ}$ for the thicker polymer films prepared, from the (1:0) polymer solutions. (b) GISAXS patterns with $\alpha_{i}=0^{\circ}$ for PPA (1:2) and PPS (1:1) films at $30{ }^{\circ} \mathrm{C}$, above the melting temperature $\left(100{ }^{\circ} \mathrm{C}\right)$ and after subsequent cooling down to $30^{\circ} \mathrm{C}$.

(Figure 7a) obtained with $\alpha_{i}=0$ lack of both a well-defined reflection peak and of well-defined Yoneda peaks indicating that most of the contribution arises from transmission rather than from reflection effects. This fact was corroborated by experiments using a bulk polyethylene Lupolen standard placed at $\alpha_{i}=0$ where the characteristic long-spacing of bulky Lupolen was detected. Figure 7a presents the GISAXS at zero incidence angle patterns obtained for the thicker polymer coatings. Besides the continuous scattering a clear out-of-meridian maximum of different shape is resolved for the three thicker samples. The maxima tend to be vertically elongated although in some cases, like for example for PPA (1:0), the maximum exhibits a curvature. The correlation length derived from these out-of-meridian maxima is $\approx 50 \mathrm{~nm}$ and is very similar for the three polymers. The shape of these patterns is characteristic of a scattering system consisting of spatially correlated scatters mostly oriented in the vertical direction. ${ }^{21}$ On a first approach and on the basis of the AFM images (Figure 2) we can identify the scatters with edge-on crystal lamellae.

Figure $5 \mathrm{~b}$ shows in a common plot the power spectral density function derived from the AFM image and the GISAXS at zero incidence angle scattering intensity ${ }^{18,19}$ of the PPA (1:1) sample. Both functions exhibit a maximum at a very similar scattering vector. The correlation length derived by GISAXS $\left(\alpha_{i}=0\right)$ from the maximum is $\approx 50 \mathrm{~nm}$ while that derived by the PSD function is $\approx 40 \mathrm{~nm}$. These two values are in rather good agreement as frequently observed in thin films containing homogeneous correlated motives. ${ }^{19}$ At this point it is worth to remember that AFM provides mainly local images of the film surface while GISAXS at $\alpha_{i}=0$ aims to provide average structural information of the film volume. Accordingly, the different correlation lengths observed by AFM and GISAXS can be attributed to a quantitative structural difference between the volume and the surface of the films. However, qualitatively both techniques indicate a significant presence of edge-on crystal lamellae.

GISAXS at $\alpha_{i}=0$ experiments as a function of temperature were also performed. Figure $7 \mathrm{~b}$ shows representative patterns obtained for the PPA (1:2) and PPS (1:1) films during a heating-cooling cycle. From these patterns it is evident the disappearance of the out-of-meridian scattering maxima at temperatures higher than the melting ones. After cooling from the molten state the original GISAXS at $\alpha_{i}=0$ pattern, with the outof-meridian reflections, is essentially recovered. This effect seems to indicate that the formation of edge-on crystal lamellae is a characteristic of these polymer thin films and not induced by the spin-coating procedure.

Finally, Figure $6 \mathrm{~b}$ shows the GIWAXS at $\alpha_{i}=0$ pattern obtained for PPA (1:0) sample, after its rotation of $90^{\circ}$ for a better comparison. Indeed, at ID13 the sample plane was placed vertically and the microfocus beam goes throughout the polymer film and parallel to the sample plane. It is remarkable the simplicity of this pattern in comparison to the GIWAXS pattern of Figure 6a (left). The GIWAXS at $\alpha_{i}=0$ pattern of PPA (1:0) will be very helpful to clarify the texture of crystallites building up the polymer films as it will be discussed in the next paragraph.

3.2.3. Wide Angle X-ray Scattering: Crystal Unit Cell Parameters and Chain Orientation of the Polymer Thin Films. To our knowledge crystal unit cell data for PPA, PPG and PPS have not yet been reported. However a great information about the crystalline structure of several aliphatic polyesters $(X, Y)$ where the glycol moiety $(X)$ has an even number of carbon atoms is available for long time ago and summarized in a recent article. ${ }^{26}$ Crystallographic data for the polyester $(3,8)$ are also reported. ${ }^{27}$ Generally these polyesters show polymorphism and present monoclinic and orthorhombic crystal phases being the monoclinic one the most stable. ${ }^{26-30}$ In order to obtain information about the crystal chain packing of the polymers investigated we performed wide-angle X-ray experiments in drawn filaments. Thin polymer filaments were drawn from the molten polymer and solidified over a Kapton foil. In Figure 8 we present the WAXS patterns obtained for the drawn polymers after subtraction of a Kapton foil pattern. The WAXS patterns exhibit significant orientation. For PPG and PPA, the persistent reflection closer to the center belongs to the Kapton foil used which appears to be inhomogeneous. In the case of PPS, the Kapton reflection is properly subtracted. The orientation, although small, is enough to make a distinction between independent reflections. On one hand, it is common for polymer systems that the strong reflections of diffractograms and 2D-patterns are related to the inter chain distances. Then, $a, b$ parameters of the crystal unit cell could be derived from such reflections. On the other hand, reflections of higher spacing values should be related to the chain unit length ( $c$ axis). Looking at the oriented patterns of Figure 8 we see that the strong reflections are not orthogonal to each other and therefore we can assume the monoclinic crystal phase to describe the chain packing in these polyesters. In addition, we can also assume a monoclinic chain packing which contains two polymer chains per crystal unit cell like that of other aliphatic polyester. ${ }^{31,32}$ Under this hypothesis, we have indexed the first strong reflection as the 020 for all three polymers. The parameter $b=2 d_{020}$ where $d_{020}$ is the lattice spacing of the reflection 020 . The $b$ axis is also considered as the principal axis of the monoclinic unit cell. This means that $b$ axis is perpendicular to both $a$, and $c$ axes

(26) Pan, P.; Inoue, Y. Prog. Polym. Sci. 2009, 34, 605-640.

(27) Jourdan, N.; Deguire, S.; Brisse, F. Macromolecules 1995, 28 8086-8091

(28) Minke, R.; Blackwell, J. J. Macromol. Sci.-Phys. 1979, B16, 407-417.

(29) Gan, Z. H.; Abe, H.; Doi, Y. Macromol. Chem. Phys. 2002, 203, 2369-2374.

(30) Pouget, E.; Almontassir, A.; Casas, M. T.; Puiggali, J. Macromolecules 2003, 36, 698-705.

(31) Turnerjones, A.; Bunn, C. W. Acta Crystallogr. 1962, 15, 105

(32) Ichikawa, Y.; Kondo, H.; Igarashi, Y.; Noguchi, K.; Okuyama, K.; Washiyama, J. Polymer 2000, 41, 4719-4727. 

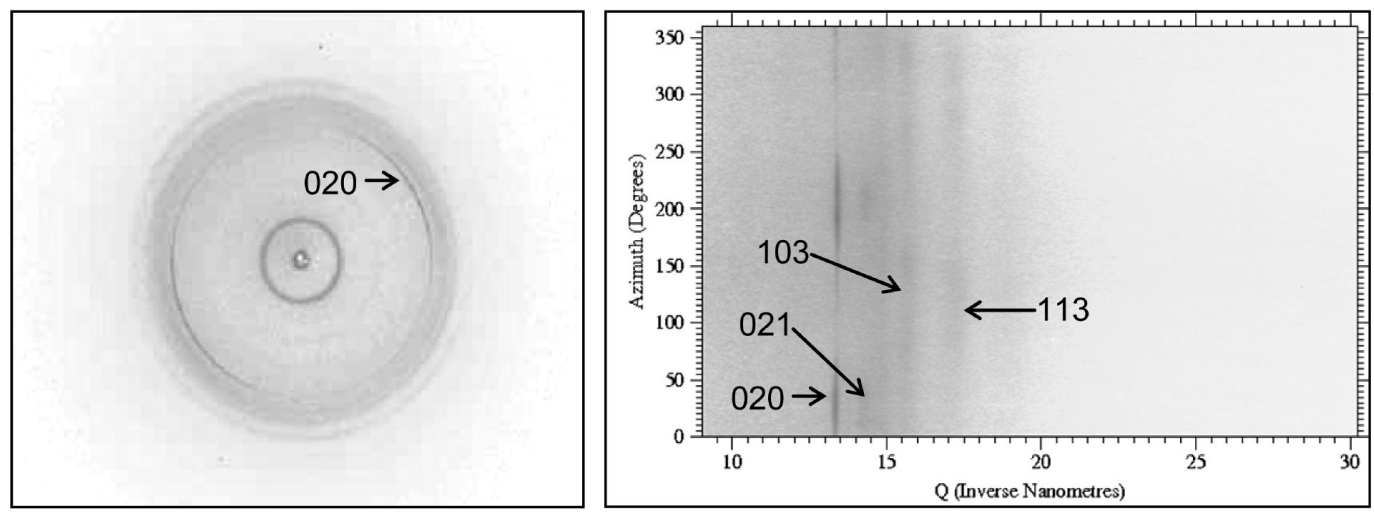

\section{PPG}
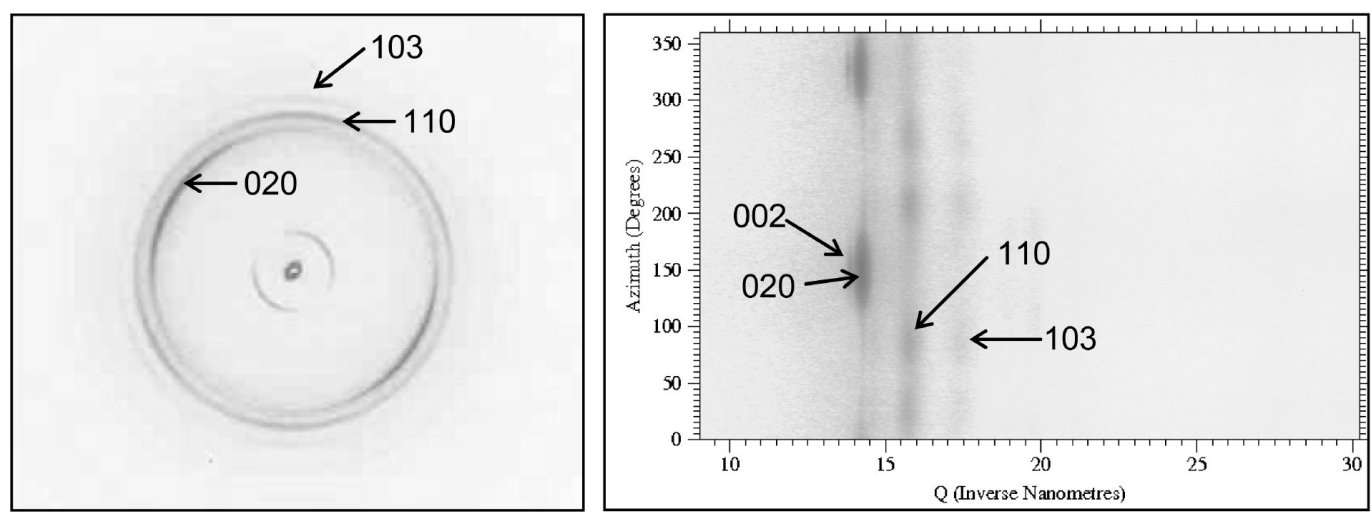

\section{PPS}
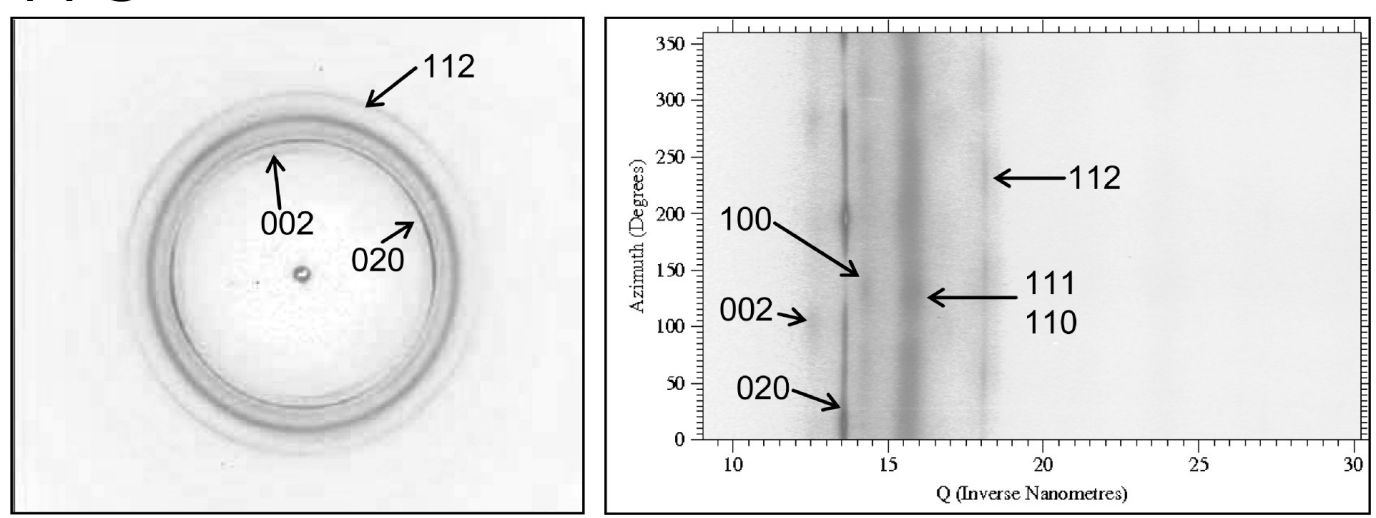

Figure 8. WAXS patterns for a melt solidified filament of the three polymers obtained with a micro beam at the ID13 beamline at the ESRF. The most inner ring corresponds to the Kapton contribution. On the right the complete azimuth projection corresponding to an interval of the scattering vector $q\left(\mathrm{~nm}^{-1}\right)$ between 8 and $30 \mathrm{~nm}^{-1}$. Crystallographic indices $h k l$ of reflections are indicated.

which define the $\beta$ angle. The lattice spacing for such a monoclinic unit cell can be calculated by the expression: ${ }^{33}$

$$
\frac{1}{d_{h k l}}=\frac{1}{\sin \beta} \sqrt{\frac{h^{2}}{a^{2}}+\frac{k^{2} \sin ^{2} \beta}{b^{2}}+\frac{l^{2}}{c^{2}}-\frac{2 h l \cos \beta}{a c}}
$$

In Table 2, we present the unit cell parameters proposed for the three polymers investigated. The last column of Table 2 corresponds to the molecular cross-section calculated according to the

(33) Cullity, B. D. Elements of X-ray diffraction; Addison-Wesley: Boston, MA, 1956.
Table 2. Crystal Unit Cell Parameters and Polymer Chain CrossSection, $A={ }^{1} / 2 b a \sin \beta$ for the Aliphatic Polyesters Investigated

\begin{tabular}{lccccc}
\hline polymer & $a(\mathrm{~nm})$ & $b(\mathrm{~nm})$ & $c(\mathrm{~nm})$ & $\beta(\mathrm{deg})$ & $A\left(\mathrm{~nm}^{2}\right)$ \\
\hline PPS & 0.4493 & 0.9254 & 1.0500 & 75 & 0.201 \\
PPG & 0.5493 & 0.8856 & 1.1000 & 55 & 0.199 \\
PPA & 0.5067 & 0.9420 & 1.2600 & 57 & 0.200
\end{tabular}

expression: $A=d_{020} d_{100}={ }^{1} / 2 b a \sin \beta$. For the three polymers, $A$ appears to be very similar as it would be expected. The corresponding hkl indexes of the observed reflections are indicated in the pattern of Figure $6 \mathrm{~b}$ and 8 . It is remarkable the very weak intensity of reflections $(001)$ which are related to the unit chain 
length. This would indicate a high disorder along the polymer chain direction or $c$-axis. Taking into account the indexing of reflections on the GIWAXS at $\alpha_{i}=0$ pattern from the spin coated sample PPA (1:0) (Figure 6b) we can now postulate that crystallites lie on the substrate with their $b c$ face parallel to the substrate plane. This indicates that the polymer chains of crystal lamellae run parallel to the substrate. We think this crystal orientation is also present for PPG and PPS thin films. Indeed, in case of PPG this is clearly supported by the great similarity between the GIWAXS (Figure 6a) and the oriented WAXS pattern (Figure 8). In the case of PPS, the strong reflection 020 observed in the oriented pattern (Figure 8) should be also located on the equator of its GIWAXS pattern (Figure 6a). Taking into account the orientation of the polymer chain in the crystal unit cell proposed for related aliphatic polyesters, ${ }^{31}$ the carbonyl groups $(\mathrm{C}=\mathrm{O})$ lie on the $b c$ plane. Thus it is plausible to think that the molecular interaction of $\mathrm{C}=\mathrm{O}$ groups with the hydrophilic nature of the outer silicon oxide layer of the substrate is controlling the chain fixation to the substrate and as a result of it the further polymer crystallization by developing edge-on lamellae preferentially.

\section{Conclusions}

Lamellar crystal edge-on morphology seems to be a rather robust characteristic of thin films of poly(propylene succinate) (PPS), poly(propylene glutarate) (PPG) and poly(propylene adipate) (PPA) prepared by spin-coating over silicon substrates. This main conclusion is in agreement with Monte Carlo simulations ${ }^{10}$ suggesting that for polymers deposited over slippery walls the edge-on lamellar crystal morphology would be the most favorable.

Acknowledgment. The authors express thanks for the financial support from the MICINN (Grants MAT2009-07789 and FPI BES-2006-12807), Spain. The experiments performed at BW4 in HASYLAB (DESY) were supported by the European Community (Contract RII3-CT-2004-506008 (IA-SFS)). We thank S. Roth and A. Timann for their support during measurements at HASYLAB. We also thank O. Bikondoa, P. Böseke (ID01), and R. Davies (ID13) for their support during measurements at ESRF. 